\title{
Nonlinear Model Reduction of a Two-Dimensional MCFC Model With Internal Reforming
}

\author{
M. Mangold*, M. Sheng \\ Max-Planck-Institut für Dynamik komplexer technischer Systeme, \\ Sandtorstraße 1, D-39106 Magdeburg, Germany \\ *Corresponding author. Tel. +49 3916110 361; fax +49 3916110 513; \\ email mangold@mpi-magdeburg.mpg.de
}

\begin{abstract}
A reduced nonlinear model of a planar molten carbonate fuel cell is presented. The model is derived from a spatially distributed dynamic model of the cell by applying the Karhunen Loève Galerkin procedure. The reduced model is of considerably lower order than the original one and requires much less computation time. The comparison between the two models shows that the reduced model can describe the dynamic of the temperature field with sufficient accuracy and has good extrapolation qualities with respect to changes in the model parameters.
\end{abstract}

Keywords: Molten carbonate fuel cell; dynamic simulation; reduced model; spatially distributed system; Karhunen- Loève decomposition 


\section{Introduction}

The molten carbonate fuel cell (MCFC) is a high-temperature fuel cell operated at $600^{\circ} \mathrm{C}-700^{\circ} \mathrm{C}$. Due to its high operation temperature, the MCFC offers advantages for the co-generation of heat and electricity. Furthermore, the high operation temperature enables internal reforming and makes the MCFC flexible with respect to the sorts of fuel that can be used. On the other hand, the operation temperatures and consequently the demands of the MCFC on thermal stability of the used materials are below those of solid oxide fuel cells [1]. Those properties make the MCFC an attractive candidate for decentralized power generation. The first commercial MCFC systems are now close to market [2].

Currently, the development and operation of MCFCs as of other high temperature fuel cells is mainly based on experimental and empirical knowledge. However, an intuitive process understanding is difficult in this case, as an MCFC system is an highly integrated process whose behavior depends on numerous interactions between the electrochemical reaction steps, the internal reforming, mass transport processes, and the heat transfer inside a cell or stack. A deeper understanding of the physico-chemical processes in an MCFC can be obtained from mathematical models based on physical conservation principles. Model based process control and process design strategies can lead to a much better use of the fuel cells' capacities and increase the efficiency of the system, but require suitable dynamic process models. Such models should offer good extrapolation qualities on the one hand, but must also be 
solvable in the framework of a real-time process control environment or an optimization algorithm. Today, the majority of MCFC models available in open literature are steady state models and therefore not suitable for process control (a survey is given in [3]). Only very few detailed dynamic MCFC models have been published $[4,5,6]$. Those models consist of systems of algebraic and nonlinear partial differential equations in several space coordinates, which are too complex for many process control purposes. As a consequence, most control studies of the MCFC have to rely on rather simple dynamical models, black-box type input output models, or qualitative knowledge-based approaches [7,8,9,10].

The purpose of this contribution is to derive a reduced nonlinear dynamic model of an MCFC that approximates a detailed spatially distributed model with good accuracy, but whose solution requires less computation time. In the next section, the detailed two-dimensional spatially distributed model of a planar MCFC with cross flow is derived from energy, mass, and charge balances. This model is used as a starting-point and reference for the reduced model. A reduced set of model equations is developed by applying the Karhunen-Loève Galerkin to the reference model. Finally, the reduced model is validated in test simulations by comparison with the reference model. 


\section{The Spatially distributed MCFC model}

For the development of a spatially distributed reference model, a planar MCFC as shown in Fig. 1 is considered. The flows an anode and on cathode side are orthogonal to each other, thereby inducing a cross flow. At the anode, a reaction between $H_{2}$ and carbonate ions $\mathrm{CO}_{3}^{2-}$ takes place that releases two electrons. The carbonate ions are produced at the cathode in a reaction of carbon dioxide and oxygen under consumption of two electrons. The transport of carbonate ions through the electrolyte

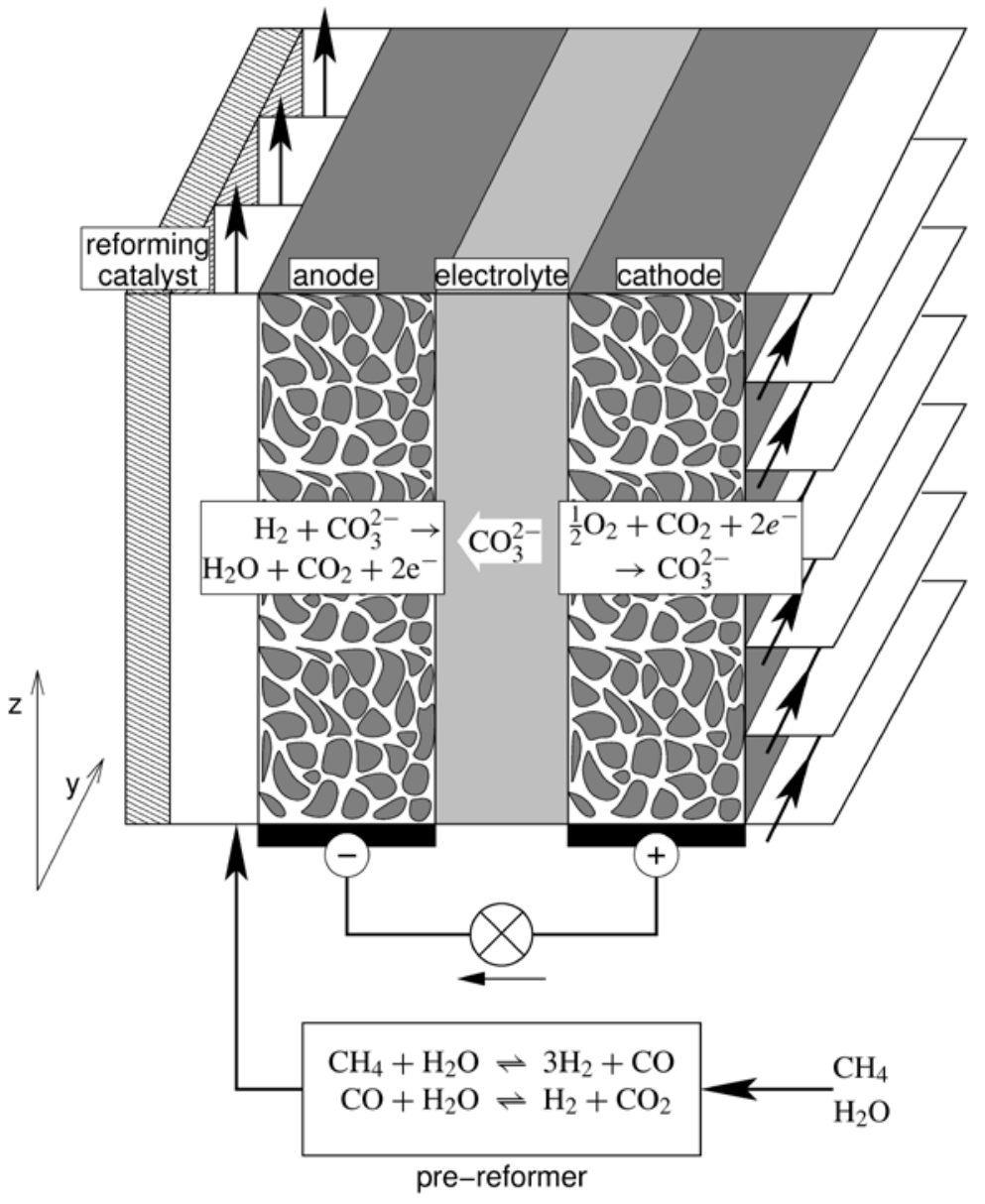

Figure 1: Basic MCFC Structure with internal reforming.

closes the internal electrical circuit. The hydrogen consumed on the anode side is generated in a steam reforming reaction. The MCFC offers different possibilities of 
external reforming, direct internal reforming inside the cell, and indirect internal reforming in connection pipes between different cells [11]. In this work, a combination of external reforming in a pre-reformer and of direct internal reforming is considered. The pre-reformer is fed with steam and methane.

In the following, a dynamic two-dimensional model of the process will be developed, which includes energy, mass, and charge balances. The main purpose of the model is to serve as a basis for the temperature control of the cell. As the dynamics of the temperature equations is much slower than that of the mass and charge balances, only the temperature equations is considered to be dynamic, the mass and charge balances are assumed to be at steady state.

\subsection{Pre-reformer}

In the pre-reformer, the following two reactions take place:

- The reforming reaction

$\mathrm{CH}_{4}+\mathrm{H}_{2} \mathrm{O} \stackrel{r_{R}}{\rightleftarrows} \mathrm{CO}+3 \mathrm{H}_{2}$

- The water-gas shift reaction

$\mathrm{CO}+\mathrm{H}_{2} \mathrm{O} \stackrel{r_{W}}{\rightleftarrows} \mathrm{CO}_{2}+\mathrm{H}_{2}$

For the description of the reformer, several assumptions are made:

- The temperature is constant, no energy balance is considered.

- The reactants behave like ideal gases.

- The following reaction kinetics from [12] is used. 


$$
\begin{gathered}
r_{R}=\frac{k^{R} \exp \left(-\frac{E_{R}}{R T}\right)\left(P_{\mathrm{CH}_{4}} P_{\mathrm{H}_{2} \mathrm{O}}^{0.5} / P_{\mathrm{H}_{2}}^{1.25}\right)\left(1-\left(P_{\mathrm{CO}} P_{\mathrm{H}_{2}}^{3} / K_{\mathrm{P}_{1}} P_{\mathrm{CH}_{4}} P_{\mathrm{H}_{2} \mathrm{O}}\right)\right)}{(\text { den })^{2}} \\
r_{W}=\frac{k^{w} \exp \left(-\frac{E_{W}}{R T}\right)\left(P_{\mathrm{CH}_{4}} P_{\mathrm{H}_{2} \mathrm{O}}^{0.5} / P_{\mathrm{H}_{2}}^{0.5}\right)\left(1-\left(P_{\mathrm{CO}_{2}} P_{\mathrm{H}_{2}} / K_{P_{2}} P_{C O} P_{\mathrm{H}_{2} \mathrm{O}}\right)\right)}{(\text { den })^{2}}
\end{gathered}
$$

where

$$
\begin{aligned}
\text { den } & =1+K_{\mathrm{CO}} P_{\mathrm{CO}}+K_{\mathrm{CO}_{2}} P_{\mathrm{CO}_{2}}+K_{\mathrm{H}} P_{\mathrm{H}}^{0.5}+K_{\mathrm{H}_{2} \mathrm{O}}\left(P_{\mathrm{H}_{2} \mathrm{O}} / P_{\mathrm{H}_{2}}\right) \\
& +K_{\mathrm{CH}_{4}}\left(P_{\mathrm{CH}_{4}} / P_{\mathrm{H}_{2}}\right)+K_{\mathrm{CHO}}\left(P_{\mathrm{CH}_{4}} P_{\mathrm{H}_{2} \mathrm{O}} / P_{\mathrm{H}_{2}}^{2.5}\right)
\end{aligned}
$$

and $P_{i}$ stands for the partial pressure of component $i$

- The pre-reformer is assumed to be one-dimensional.

The following component material balances result:

$$
-\frac{\partial\left(\dot{n} X_{i, r}\right)}{\partial z}+v_{R i} r_{R}+v_{w i} r_{w}=0, \quad i=\mathrm{CH}_{4}, \mathrm{H}_{2} \mathrm{O}, \mathrm{CO}, \mathrm{CO}_{2}, \mathrm{H}_{2}
$$

In the above equation, $X_{i, r}$ is the molar fraction of the gas components in the pre-reformer; $\dot{n}$ is the molar flow rate, $v_{R i}$ and $v_{w i}$ are the stoichiometric coefficients.

For the total material balance, one obtains:

$$
-\frac{\partial \dot{n}}{\partial z}+2 r_{R}=0
$$

\subsection{Model of the MCFC}

The model of the MCFC is based on the following assumptions:

- The electrodes' area is described through a y-z plane. The gas flow on anode side is in $\mathrm{z}$ direction, and the gas flow on the cathode side is in y direction.

- Spatial gradients of the concentrations and the temperature in solely $\mathrm{y}$ or $\mathrm{z}$ 
direction are considered. Gradients perpendicular to the $\mathrm{y}-\mathrm{z}$ plane are neglected.

- For the sake of simplicity, a pseudo-homogeneous energy balance is derived, i.e. temperature differences between the gases and the solid parts of the cell are neglected.

- The anodic reaction

$\mathrm{H}_{2}+\mathrm{CO}_{3}^{2-} \rightarrow \mathrm{H}_{2} \mathrm{O}+\mathrm{CO}_{2}+2 e^{-}$

and the cathodic reaction

$\frac{1}{2} \mathrm{O}_{2}+\mathrm{CO}_{2}+2 e^{-} \rightarrow \mathrm{CO}_{3}^{2-}$

are taken into account. To calculate their reaction rates, the Butler-Volmer approach is used. For the reforming and the shift reaction (1) and (2), the kinetics by [12] is used, as in the pre-reformer model.

- The potentials of the electrodes are assumed to be spatially independent. Ohmic losses are modeled by an average Ohmic resistance for the cell.

- The mass and charge balances are assumed to be quasi-stationary, as their time constants are very small compared with the time constant of energy balance.

The above assumptions lead to the balance equations summarized in the following.

\subsubsection{Energy balance of the MCFC}

The energy balance in the form of a parabolic partial differential equation of the temperature has the following structure: 


$$
\begin{aligned}
0= & \left(\rho C_{P}\right) d \frac{\partial T}{\partial t}+\dot{n}^{A} C_{P}^{A} \frac{\partial T}{\partial z}+\dot{n}^{C} C_{P}^{C} \frac{\partial T}{\partial y}-\lambda d\left(\frac{\partial^{2} T}{\partial y^{2}}+\frac{\partial^{2} T}{\partial z^{2}}\right)+\left(\Phi^{A M}-\Phi^{A C}\right) i^{A} \\
& +\left(\Phi^{C C}-\Phi^{C M}\right) i^{C}-\frac{i^{A}}{2 F}\left(-\Delta h_{R}^{A}\right)+\frac{i^{C}}{2 F}\left(-\Delta h_{R}^{C}\right)-r_{R}\left(-\Delta h_{R}^{R}\right)-r_{W}\left(-\Delta h_{R}^{W}\right) \\
& -\frac{I_{\text {Cell }}}{L_{y} L_{z}}\left(\Phi^{A M}-\Phi^{C M}\right)=: \operatorname{Re} s
\end{aligned}
$$

The boundary conditions read:

$$
T(t, y, 0)=T_{i n}^{A}(t, y) \quad T(t, 0, z)=\left.T_{i n}^{C}(t, z) \quad \frac{\partial T}{\partial z}\right|_{t, y, L_{z}}=\left.0 \quad \frac{\partial T}{\partial y}\right|_{t, L_{y}, z}=0
$$

In the above equations, $\rho c_{p}$ is the total thermal capacity of the system; $d$ is a geometrical constant comprising the thickness of anode, cathode, and membrane; $\dot{n}^{A}$, $\dot{n}^{C}$ are the molar flow rates of anode, and cathode gas flow, respectively; $C_{P}^{A}, C_{P}^{C}$ are molar heat capacities of anode, and cathode gas flow, respectively; $\lambda$ is the heat conductivity; $i^{A}, i^{C}$ are the electrical current densities of anode, and cathode; $\Delta h_{R}^{A}$, $\Delta h_{R}^{C}, \Delta h_{R}^{R}, \Delta h_{R}^{W}$ are the heats of reaction of reaction (5), (6), (1) and (2), respectively; $\Phi^{A C}, \Phi^{C C}$ are the electrical potentials of the anode and the cathode; $\Phi^{A M}, \Phi^{C M}$ are the electrical potentials of the electrolyte membrane on anode side and on cathode side; $I_{\text {Cell }}$ is the total cell current; $L_{y}, L_{z}$ are the lengths of the electrodes in y and $\mathrm{z}$ directions; $F$ is the Faraday constant.

\subsubsection{Reaction kinetics}

- Anode side

$$
i^{A}=k_{0}^{A} \exp \left(-\frac{E^{A}}{I R T}\right)\left\{\exp \left(\frac{(1-\beta) 2 F}{I R T} \eta^{A}\right) \cdot \frac{x_{H_{2}}^{A}}{x_{H_{2, \text { ref }}}^{A}}-\exp \left(-\frac{\beta 2 F}{I R T} \eta^{A}\right) \cdot \frac{x_{\mathrm{H}_{2} \mathrm{O}}^{A} x_{\mathrm{CO}_{2}}^{A}}{x_{\mathrm{H}_{2} \mathrm{O}, \text { ref }}^{A} x_{\mathrm{CO}_{2} \text { ref }}^{A}}\right\}
$$

- Cathode side 


$$
i^{C}=-k_{0}^{C} \exp \left(-\frac{E^{C}}{I R T}\right)\left\{\exp \left(\frac{(1-\beta) 2 F}{I R T} \eta^{C}\right)-\exp \left(-\frac{\beta 2 F}{I R T} \eta^{C}\right) \cdot \sqrt{\frac{x_{O_{2}}^{C}}{x_{O_{2} \text { ref }}^{C}}} \frac{x_{\mathrm{CO}_{2}}^{C}}{x_{\mathrm{CO}_{2} \text { ref }}^{C}}\right\}
$$

The over-potential $\eta^{A}$ and $\eta^{C}$ are given by:

$$
\begin{aligned}
& \eta^{A}:=\underbrace{\left(\Phi^{A C}-\Phi^{A M}\right)}_{\Delta \Phi^{A}}-\left(\Phi^{A C}-\Phi^{A M}\right)_{r e f} \\
& \eta^{C}:=\underbrace{\left(\Phi^{C C}-\Phi^{C M}\right)}_{\Delta \Phi^{C}}-\left(\Phi^{C C}-\Phi^{C M}\right)_{r e f}
\end{aligned}
$$

$\left(\Phi^{A C}-\Phi^{A M}\right)_{\text {ref }}$ and $\left(\Phi^{C C}-\Phi^{C M}\right)_{\text {ref }}$ are the equilibrium potentials of the anode reaction and the cathode reaction, respectively.

\subsubsection{Material balances}

- Component and total material balances on the anode side

$$
\begin{aligned}
& 0=-\frac{\partial\left(\dot{n}^{A} \cdot x_{i}^{A}\right)}{\partial z}+v_{A, i} \frac{i^{A}}{2 F}+v_{R i} r_{R}+v_{w i} r_{W} \\
& 0=-\frac{\partial \dot{n}^{A}}{\partial z}+\frac{i^{A}}{2 F}+2 r_{R}
\end{aligned}
$$

- Component and total material balances on the cathode side

$$
\begin{aligned}
& 0=-\frac{\partial\left(\dot{n}^{C} x_{i}^{C}\right)}{\partial y}-v_{C, i} \frac{i^{c}}{2 F} \\
& 0=-\frac{\partial \dot{n}^{C}}{\partial y}-\frac{3 i^{c}}{4 F}
\end{aligned}
$$

\subsubsection{Charge balances}

The current densities on anode and on cathode side must sum up to the cell current

$I_{\text {Cell }}$ :

$$
I_{\text {cell }}=\int_{0}^{L_{Y}} \int_{0}^{L_{z}} i^{A} d y d z
$$$$
\text { (15) } \quad I_{\text {cell }}=\int_{0}^{L_{y}} \int_{0}^{L_{z}} i^{C} d y d z
$$ 
The total cell voltage is given by:

$\left(\Phi^{C C}-\Phi^{A C}\right)=\left(\Phi^{A M}-\Phi^{A C}\right)_{r e f}-\left(\Phi^{C M}-\Phi^{C C}\right)_{r e f}-\eta^{A}+\eta^{C}-\frac{I_{\text {cell }}}{\sigma^{M}} \frac{d_{M}}{L_{Y} L_{Z}}$

where $d_{M}$ is the thickness of the electrolyte membrane, and $\sigma^{M}$ is the electrolyte's conductivity.

\subsection{Simulation results}

Typical simulation results for the temperature and current density profiles are shown in Fig.2 and Fig.3. Fig. 2 shows steady state results for a MCFC fed with hydrogen. No internal reforming takes place. The exothermic electrochemical reaction causes a temperature increase in the cell. As the cell is cooled only by the incoming gas flows, the temperature maximum lies in the corner opposite to the anode and cathode gas inlet - the upper right corner of the temperature diagram in Fig.2 (a). The reaction rates increase with increasing temperature, but decrease with increasing consumption of the reactants. Therefore, the maximum of the current density is shifted to the left with respect to the temperature maximum.

The temperature and current density profiles change if the cell is fed with methane and water instead of hydrogen. In this case, which is shown in Fig. 3, the endothermic internal reforming leads to an additional cooling of the cell. The composition of the gas flow leaving the pre-former and entering the anode side is close to the equilibrium of the steam reforming reaction. Therefore, the cooling influence of the internal reforming step is only weak in the inlet area of the anode gas channels. The influence becomes stronger towards the anode outlet, because hydrogen is consumed along the 
anode gas channels and is reproduced by the reforming reaction. Consequently, the temperature gradients in the cell are smaller than they are for a cell without internal reforming.

The two examples show that spatial dependencies have an important impact on the over-all behavior of the MCFC and have to be taken into account when modeling the system. On the other hand, the numerical solution of a dynamic spatially distributed system is difficult and time consuming. Spatially distributed models often are too complex for real-time applications like model-based control strategies or online optimization. This motivates the development of reduced models that are able to approximate the behavior of the distributed model with good quality, but require less computation time.

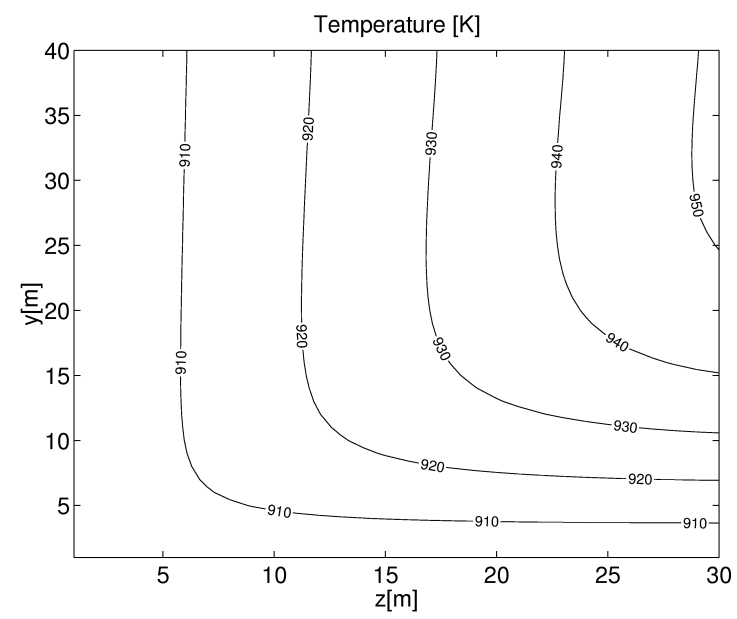

(a)

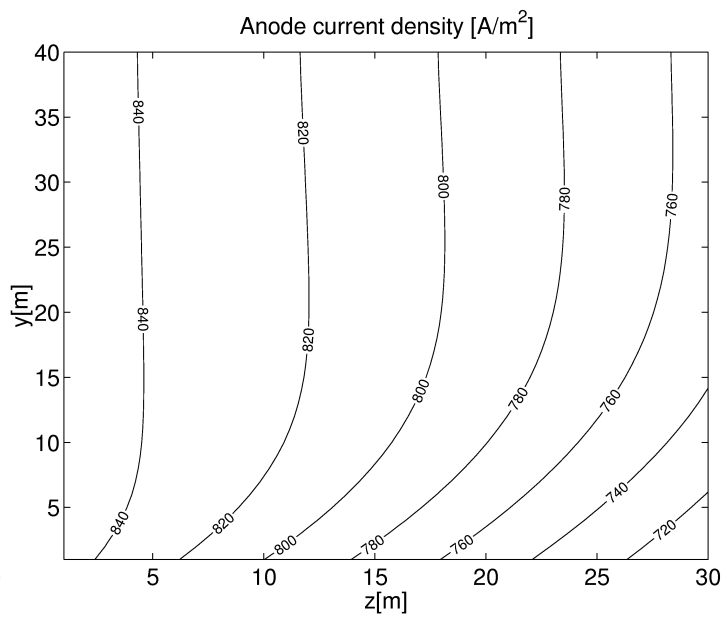

(b)

Figure 2: Steady state temperature (a) and current density profiles (b) of the model without the pre-reformer for a cell current of $I_{\text {Cell }}=200 \mathrm{~A}$; z-coordinate $=$ direction of the anode gas flow, $z=0$ being the anode gas inlet; $y$-coordinate = direction of the cathode gas flow, $y=0$ being the cathode gas inlet. 


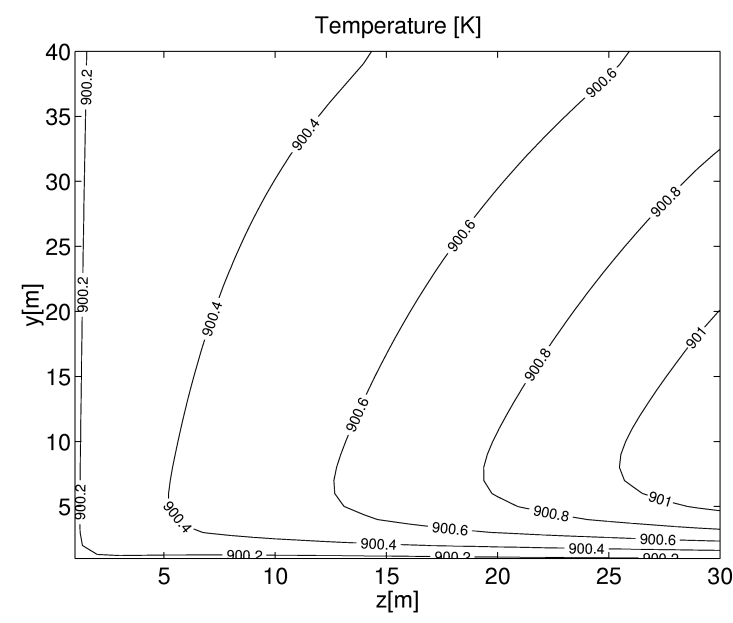

(a)

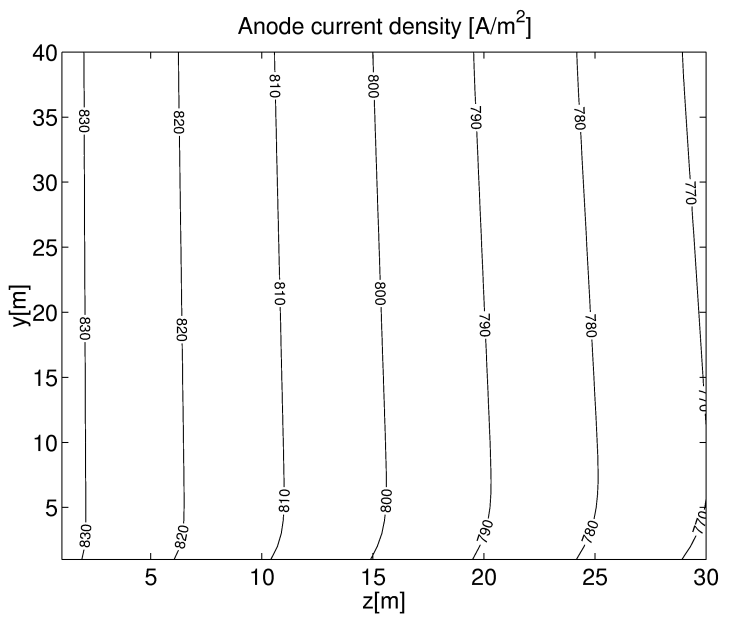

(b)

Figure 3: Steady state temperature (a) and current density profiles (b) of the model with a pre-reformer for a cell current of $I_{\text {Cell }}=200 \mathrm{~A}$; z-coordinate $=$ direction of the anode gas flow, $z=0$ being the anode gas inlet; $y$-coordinate $=$ direction of the cathode gas flow, $y=0$ being the cathode gas inlet.

\section{Derivation of the reduced MCFC model}

\subsection{Galerkin procedure}

For model reduction of parabolic partial differential equations (PDEs) like (7), orthogonal projection methods have become a frequently used technique $[13,14,15]$.

The basic idea is to represent the unknown variable, e.g. the temperature $T$, by an infinite sum of products.

$T(y, z, t)=\sum_{i=1}^{\infty} T_{i}(t) \phi_{i}^{T}(y, z)$

In (18), $\phi_{i}^{T}(y, z)$ are orthonormal basis functions with the following property: 
$\int_{y=0}^{L_{y}} \int_{z=0}^{L_{z}} \phi_{i}^{T}(y, z) \phi_{j}^{T}(y, z) d y d z= \begin{cases}0 & \text { for } i \neq j \\ 1 & \text { for } i=j\end{cases}$

The model reduction is achieved by approximating the infinite sum by a series with a finite number of elements and neglecting the higher order terms: ${ }^{1}$

$\hat{T}(x, y, t)=\sum_{i=1}^{N^{T}} \tilde{T}_{i}(t) \phi_{i}^{T}(x, y)$

In general, the approach (18) will not solve the equation (7) exactly, but a nonzero residual Res will remain. The Galerkin method of weighted residuals requires that the residual Res in (7) must vanish, if weighted by a basis function, i.e

$$
\int_{y=0}^{L_{y}} \int_{z=0}^{L_{z}} \operatorname{Res} \phi_{i}^{T} d y d z \stackrel{!}{=} 0 \quad i=1, \cdots, N^{T}
$$

This approach leads to $N^{T}$ conditions for the time dependent functions $\tilde{T}_{i}(t)$. Consequently, the partial differential equation (7) is replaced by $N^{T}$ ordinary differential equations in the reduced model.

For the model reduction of the MCFC model, not only the profile of the temperature, but also those of the molar fractions in the anode and in the cathode gas channels, as well as the profiles of the total molar flow rates have to be approximated by basis functions. Using the notation

$$
\begin{array}{ll}
\hat{x}_{j}^{A}(y, z, t):=\sum_{i=1}^{N_{j}^{A}} \tilde{x}_{j, i}^{A}(t) \phi_{i}^{A, j}(y, z) & j=C_{4}, H_{2} O, C O_{2}, H_{2}, C O \\
\hat{x}_{j}^{C}(y, z, t):=\sum_{i=1}^{N_{j}^{C}} \tilde{x}_{j, i}^{C}(t) \phi_{i}^{C, j}(y, z) & j=O_{2}, C O_{2}
\end{array}
$$

\footnotetext{
${ }^{1}$ A more accurate approximation can be obtained by using the method of approximate inertial manifolds for the higher order terms instead of neglecting them completely [16]. However, this is not considered here.
} 


$$
\begin{aligned}
& \hat{\dot{n}}^{A}:=\sum_{i=1}^{N^{n, A}} \tilde{\dot{n}}_{i}^{A}(t) \phi_{j}^{n, A}(y, z) \\
& \hat{\dot{n}}^{C}:=\sum_{i=1}^{N^{n, C}} \tilde{\dot{n}}_{i}^{C}(t) \phi_{j}^{n, C}(y, z)
\end{aligned}
$$

one obtains the following set of reduced model equations by applying the weighted residual condition to the energy and material balances:

$$
\begin{aligned}
& \left(\rho C_{P}\right) d \frac{\partial \tilde{T}_{i}}{\partial t}=\int_{y=0}^{L_{y}} \int_{z=0}^{L_{z}}\left\{-\dot{n}^{A} C_{P}^{A} \frac{\partial T}{\partial z}-\dot{n}^{C} C_{P}^{C} \frac{\partial T}{\partial y}+\lambda d\left(\frac{\partial^{2} T}{\partial y^{2}}+\frac{\partial^{2} T}{\partial z^{2}}\right)-\left(\Phi^{A M}-\Phi^{A C}\right) i^{A}\right. \\
& -\left(\Phi^{C C}-\Phi^{C M}\right) i^{C}+\frac{i^{A}}{2 F}\left(-\Delta h_{R}^{A}\right)-\frac{i^{C}}{2 F}\left(-\Delta h_{R}^{C}\right)+r_{R}\left(-\Delta h_{R}^{R}\right)+r_{W}\left(-\Delta h_{R}^{W}\right) \\
& \left.+\frac{I_{\text {Cell }}}{L_{y} L_{z}}\left(\Phi^{A M}-\Phi^{C M}\right)\right\} \phi_{i}^{T}(y, z) d y d z \\
& 0=\int_{y=0}^{L_{y}} \int_{z=0}^{L_{z}}\left\{-\frac{\partial\left(\hat{\dot{n}}^{A} \cdot \hat{x}_{j}^{A}\right)}{\partial z}+v_{A, j} \frac{i^{A}}{2 F}+v_{R j} r_{R}+v_{w j} r_{W}\right\} \phi_{i}^{A, j}(y, z) d y d z \\
& j=\mathrm{CH}_{4}, \mathrm{H}_{2} \mathrm{O}, \mathrm{CO}_{2}, \mathrm{H}_{2}, \mathrm{CO} \quad i=1, \cdots, N_{j}^{\mathrm{A}} \\
& 0=\int_{y=0}^{L_{y}} \int_{z=0}^{L_{z}}\left\{-\frac{\partial\left(\hat{\dot{n}}^{C} \hat{x}_{j}^{C}\right)}{\partial y}-v_{C, j} \frac{i^{c}}{2 F}\right\} \phi_{i}^{C, j}(y, z) d y d z \\
& j=\mathrm{O}_{2}, \mathrm{CO}_{2} \quad i=1, \cdots, N_{j}^{C} \\
& 0=\int_{y=0}^{L_{y}} \int_{z=0}^{L_{z}}\left\{-\frac{\partial \hat{\dot{n}}^{A}}{\partial z}+\frac{i^{A}}{2 F}+2 r_{R}\right\} \phi_{i}^{n, A}(y, z) d y d z \quad i=1, \cdots, N^{n, A} \\
& 0=\int_{y=0}^{L_{y}} \int_{z=0}^{L_{z}}\left\{-\frac{\partial \hat{\dot{n}}^{C}}{\partial y}-\frac{3 i^{c}}{4 F}\right\} \phi_{i}^{n, c}(y, z) d y d z \quad i=1, \cdots, N^{n, c}
\end{aligned}
$$

The charge balances (15) and (16) complete the reduced model. In summary, the reduced model is a differential algebraic system of differential index one for the unknowns $\tilde{T}_{i}, \quad \tilde{x}_{j, i}^{A}, \quad \tilde{x}_{j i}^{C}, \quad \tilde{\dot{n}}_{i}^{A}, \quad \tilde{\dot{n}}_{i}^{C}, \Delta \phi^{A}, \Delta \phi^{C}$. The quality of the reduced model, i.e. 
its deviation from the original model, mainly depends on two factors. The first one is the number of terms considered in the approximations (19), (21)-(24) . The second is the choice of the basis functions. A good approximation of the complete model by a low order reduced model is achievable, if suitable problem-specific basis functions are chosen. One possibility would be to linearize the original spatially distributed system and to use the eigenfunctions of the linearized problem as basis functions [13]. In this contribution, another approach, the Karhunen-Loève decomposition method is applied, because by that method it is also possible to incorporate the nonlinear behavior of the system in the basis functions.

\subsection{The Karhunen- Loève decomposition}

The Karhunen-Loève decomposition (K-L decomposition) was originally developed for the description of stochastic data [17]. By applying the K-L decomposition to a given stochastic field, eigenfunction are obtained that can reproduce the data with a certain accuracy and with a minimum number of degrees of freedom.

For the solution of partial differential equation, the K-L decomposition method can be used to generate basis function for the Galerkin procedure [18].

The key issue in this approach is to determine suitable basis functions from the simulation results with the original model, taken at discrete time points $t_{i}, i=1, \ldots, N$. The simulation results are called snapshots and are denoted as $v_{i}(y, z)$. By the K-L decomposition, it is possible to extract the most typical or characteristic structure from these snapshots in the form of empirical eigenfunctions $\phi_{i}(y, z)$. 
As is shown in [18], the basis functions $\phi_{i}(y, z)$ can be written as:

$\phi_{i}(y, z)=\sum_{i=1}^{N} \alpha_{i}^{T} \cdot v_{i}(y, z)$

In (29), $\alpha_{i}$ is the eigenvector of an $N \times N$ matrix $\mathrm{C}$ whose elements are given by:

$$
\begin{aligned}
& C_{i, j}^{M}=\frac{1}{N} \int_{y=0}^{L_{y}} \int_{z=0}^{L_{z}} v_{i}(y, z) v_{j}(y, z) d y d z \\
& C^{M} \alpha_{i}=\lambda_{i} \alpha_{i}
\end{aligned}
$$

For the eigenvalue $\lambda_{i}$, the following correlation holds [16]:

$$
\begin{aligned}
& \lambda_{i}=\left\langle\left(\phi_{i}, v_{n}\right)^{2}\right\rangle \\
& \left(\phi_{i}, v\right):=\int_{y=0}^{L_{y}} \int_{z=0}^{L_{z}} \phi_{i}(y, z) v(y, z) d y d z \\
& \left\langle v_{n}\right\rangle:=\frac{1}{N} \sum_{n=1}^{N} v_{n}(y, z)
\end{aligned}
$$

Therefore, $\lambda_{i}$ may be interpreted as a measure of how well an eigenfunction $\phi_{i}$ is able to approximate the time average of the snapshots. In this sense, the eigenfunction $\phi_{1}$ corresponding to the largest eigenvalue $\lambda_{1}$ is the most typical structure of the snapshots.

In order to determine suitable basis functions for the MCFC model, the response of the complete model to a increase of the cell current from 200A to 250A and subsequent decrease to $200 \mathrm{~A}$ is computed numerically by using the method of lines. The basis functions for the reduced model are computed from the transient solution of the complete model. For the temperature profile, between $N^{T}=1$ and $N^{T}=5$ basis functions are chosen. For the other variables of the reduced model, two basis function for each gas are found to be sufficient. 


\subsection{Numerical solution of the reduced model equations}

Due to the nonlinearity of the reference model and as the basis functions of the reduced model are given as data arrays, the evaluation of the integrals on the right-hand sides of Eqn. (25)-(29) requires numerical quadrature. For the MCFC model, the very simple approach to approximate the integrands by piecewise constant functions on an equidistant grid brought satisfactory results. A more crucial point for the success of the model reduction seems to be the way how the boundary conditions of the original model are included in the reduced model. Park and Cho [18] used integration in parts in order to substitute the boundary conditions into the reduced model equations. In our case, it proved to be more successful to incorporate the boundary conditions in the finite difference approximations of the temperature and concentration gradients.

\section{Simulation results of the reduced MCFC model}

Test simulations are made in order to validate the reduced model by comparson with the original model. Special emphasis is laid on the extrapolation qualities of the reduced model. The first test consists in the simulation of a randomly varying cell current. The results for a cell without internal reforming are shown in Fig. 4 It is found that already the approximation of the temperature profile by a single basis function leads to a quite satisfactory behavior of the reduced model. The temperature error becomes very small, if 5 basis functions are used for the temperature. In all simulations shown in Fig. 4, the cell voltage of the reduced model matches the result 
of the complete model nearly perfectly. The corresponding simulation for the case with internal reforming (Fig. 5) leads to similar results. Compared with Fig. 4, the temperature error of the reduced model is even smaller, mainly because the temperature gradients of the model with internal reforming are not as big as in the case without internal reforming.

A second test is the response of the reduced model to a change of the steam-to-carbon ratio in the feed. This test is more challenging than the first one, because the basis functions for the reduced model were obtained from simulations with a fixed steam-to-carbon ratio. Nevertheless, the agreement between the reduced and the complete model is still very good, as can be seen in Fig. 6. The extrapolation qualities of the reduced model could be confirmed in further tests, where the kinetic parameters of the anodic and the cathodic reaction were varied. An example is given in Fig. 7, which contains steady state results for different kinetic parameters of the anodic reaction. Although the parameters are varied over a large interval, the error of the reduced model hardly changes.

The K-L decomposition technique leads to a considerable reduction in terms of the order of the system as well as in terms of the computation time. This is illustrated for the test simulation in Fig. 5: After a spatial discretization, the complete model consists of about 12,000 equations. Its numerical solution requires about 43,000 s of a CPU time on a PC. In comparison, the reduced model consists of 25 equations, if 5 temperature basis functions are used. Its numerical solution takes about 380s of CPU time on the same PC. The decrease of the computational time achieved by the model 
reduction is not quite as strong as the decrease of the order of the system. The reason is that the evaluation of the reduced model equations is more complicated as it requires a numerical quadrature.

(a)

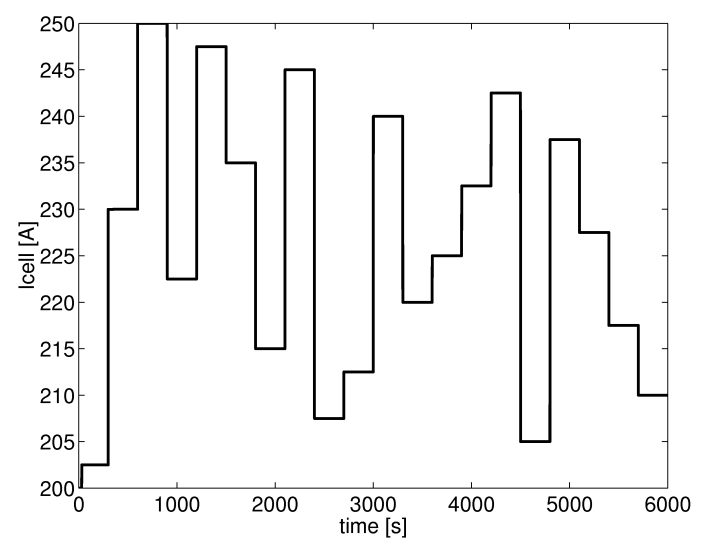

(b)

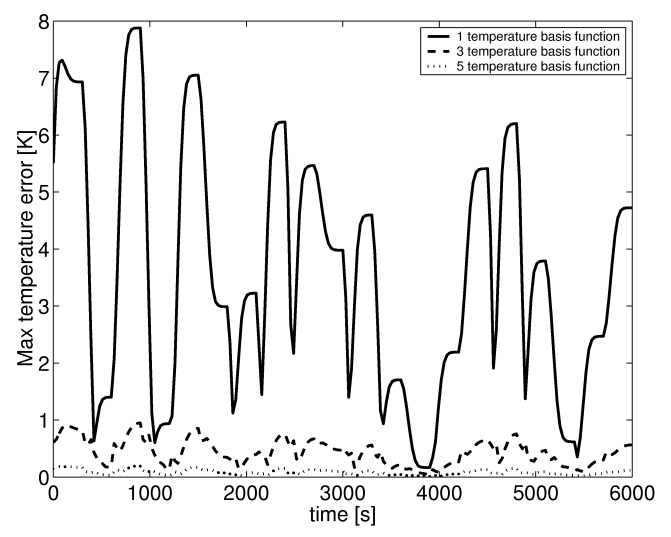

(c)

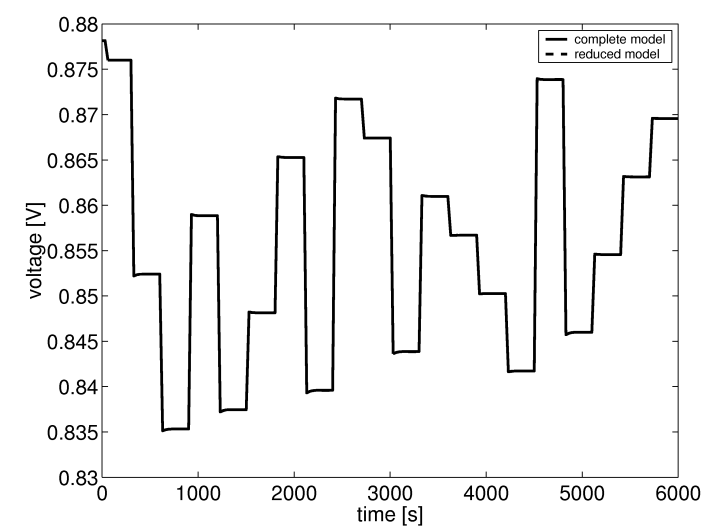

Figure 4: Validation of the reduced model by a test simulation with randomly varying cell current case without internal reforming; (a) cell current used as input signal; (b) maximum temperature error of the reduced model; (c) cell voltage of reduced and complete model. 


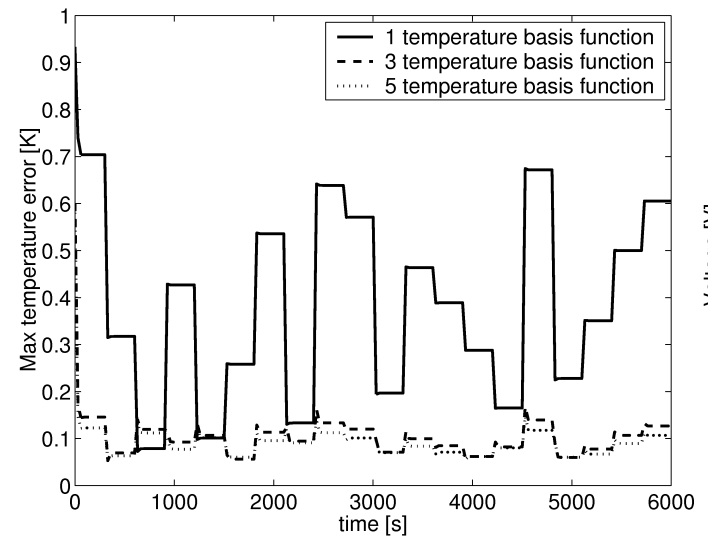

(a)

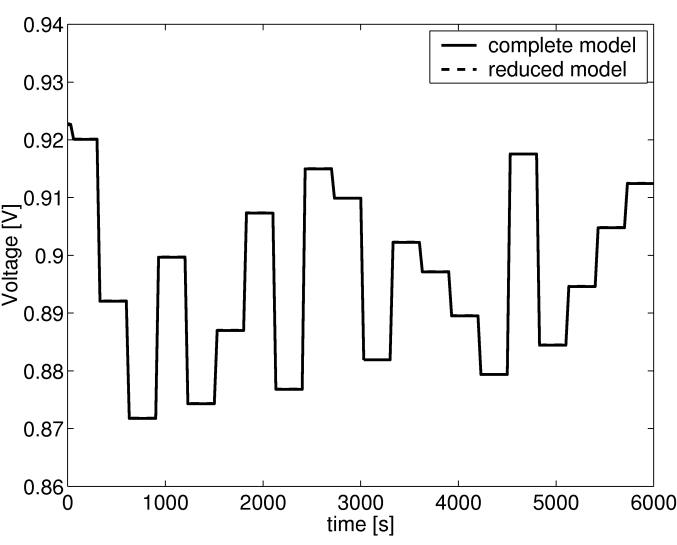

(b)

Figure 5: Validation of the reduced model by a test simulation with randomly varying cell current - case with internal reforming; cell current as in Fig. 4 (a); (a) maximum temperature error of the reduced model; (b) cell voltage of reduced and complete model.

(a)

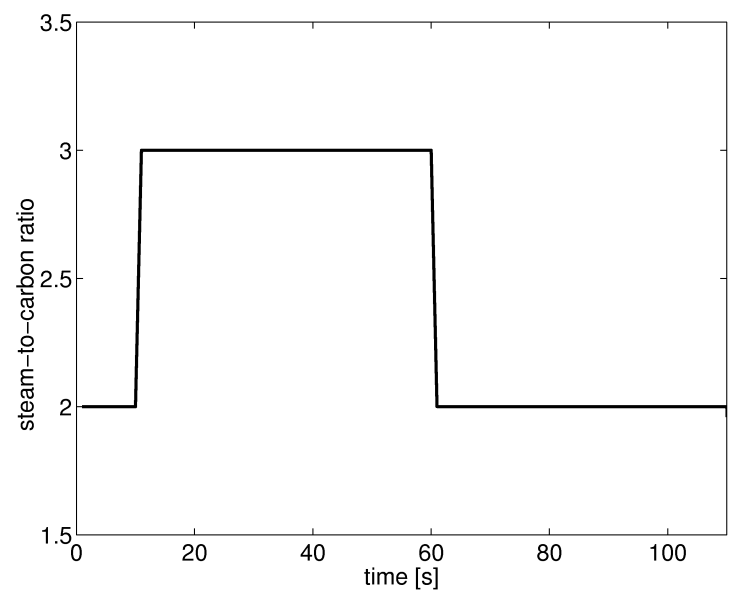

(b)

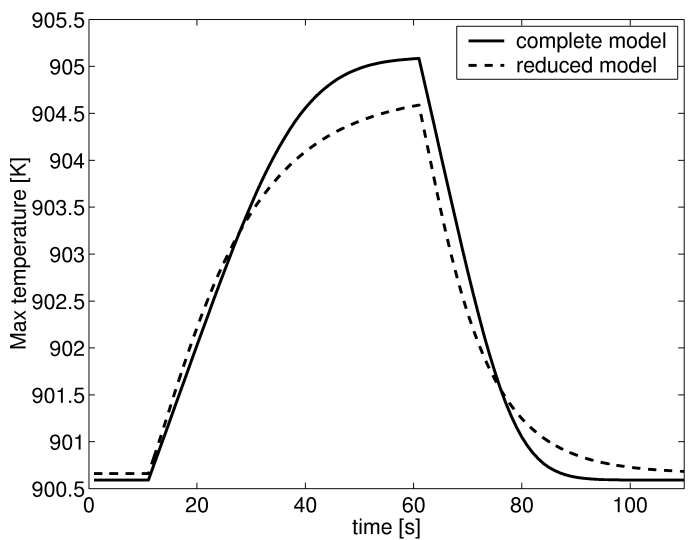

Figure 6: Validation of the reduced model by a test simulation with a varying steam-to-carbon ratio; (a) ratio of the water and methane concentration in the feed; (b) temperature error of the reduced model; (c) cell voltage of the reduced and the complete model. 
(c)

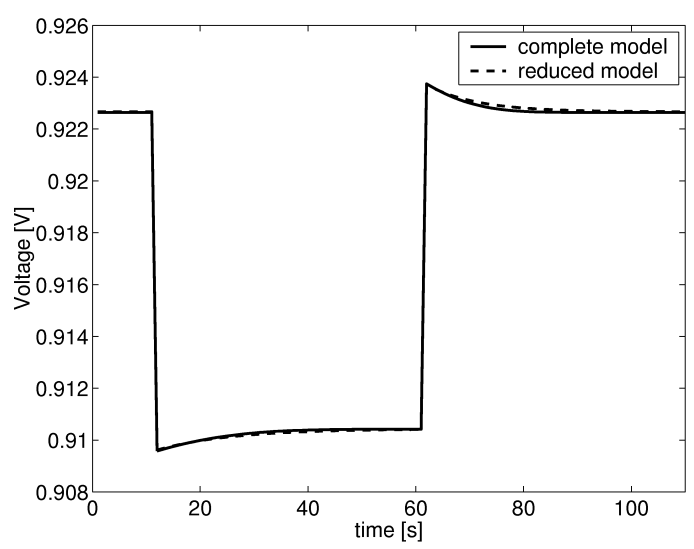

(a)

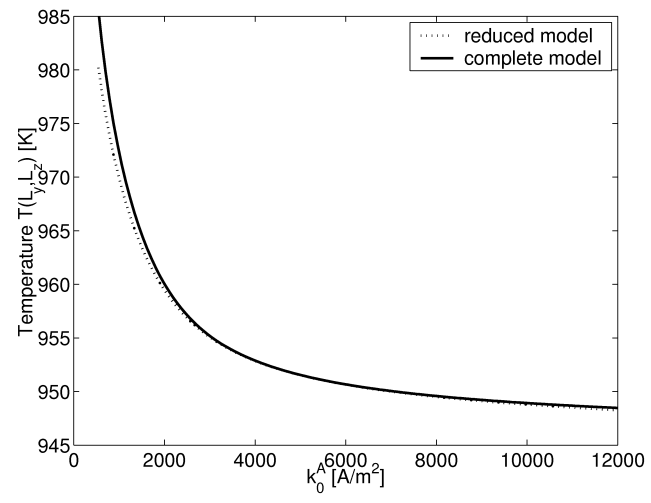

(c)

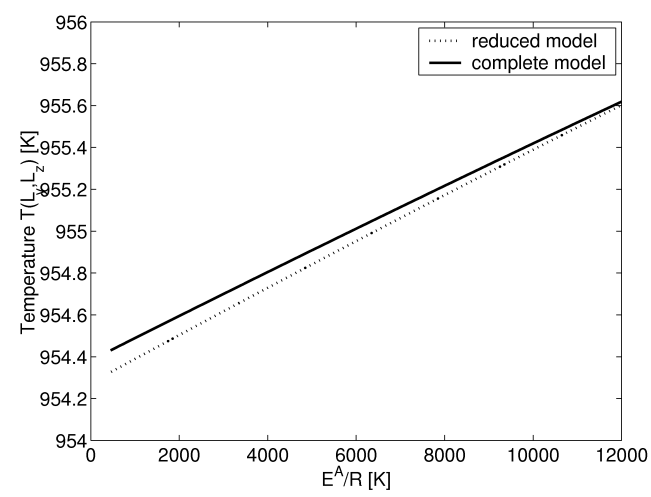

(b)

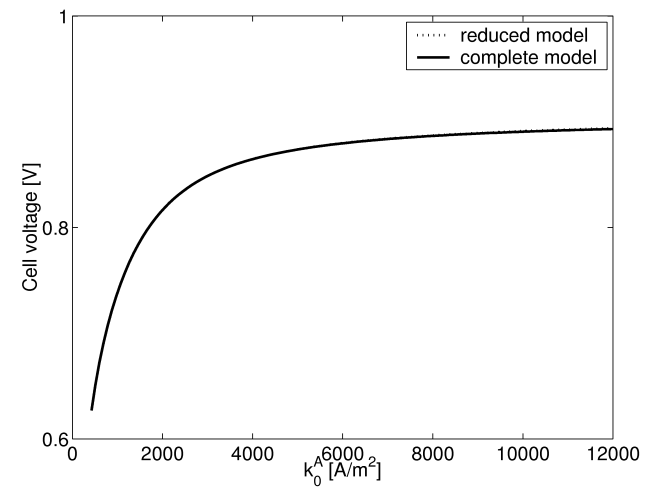

(d)

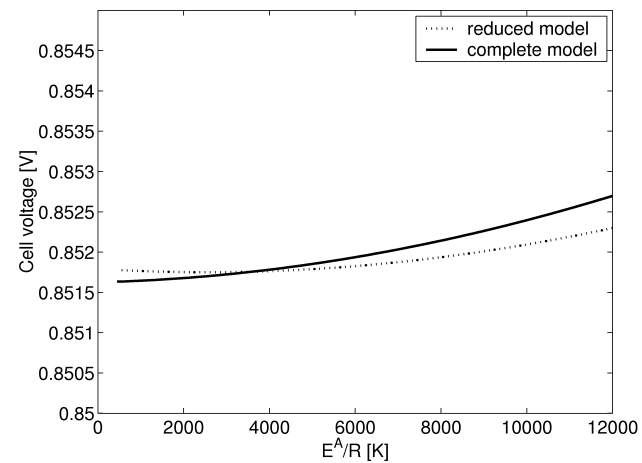

Figure 7: Validation of the reduced model by comparison of steady state results for different values of the anodic kinetic parameters; (a), (b) variation of $k_{0}^{A}$; (c), (d) variation of $E^{A}$. 


\section{Conclusions}

A reduced model of an MCFC was obtained by applying the Karhunen-Loève Galerkin method to a two-dimensional spatially distributed cross-flow model of the cell. The basic idea of the method is to approximate the profiles of the spatially distributed variables by basis functions obtained from test simulations with a detailed reference model. For the MCFC model considered here, this technique proves to be successful. The reduced model produces results that are very close to those of the original model, but it reduces the computation time by a factor of more than 100 . It may be regarded as a certain drawback of the K-L decomposition method, that the resulting eigenfunctions depend on the choice of the numerical test simulations or snapshots. However, the results of the previous section indicate satisfactory extrapolation qualities of the reduced model of the MCFC. That may be explained by the fact, that only the basis functions of the reduced model are obtained numerically. The reduced model itself is given in an analytical form and still contains the information on the physical correlations in the original model.

Due to its properties, the reduced model seems to be suitable for applications in the field of model based process control. An example are model based measuring techniques, where the reduced model can be used in the framework of a Luenberger observer or a Kalman filter in order to estimate quantities of a fuel cell that are not accessible to direct measurements. 


\section{Acknowledgements}

The financial support of the German Ministry of Education and Research under contract no. 03C0345B is gratefully acknowledged.

\section{References:}

[1] L. Carrette, K.A. Friedrich, U. Stimming, Fuel Cells 2001, 1, 5-32.

[2] M. Bischoff, G. Huppmann, Journal of Power Sources 2002, 105, 216-221.

[3] J. Koh, B. Kang, H. Lim, AIChE Journal 2001, 47, 1941-1956.

[4] W. He, Q. Chen, Journal of Power Sources 1998, 73, 182-192.

[5] P. Heidebrecht, K. Sundmacher, Chemical Engineering Science 2003, 58, 1029-1036.

[6] P. Heidebrecht, K. Sundmacher, Fuel Cells 2003, 2, 166-180.

[7] M.D. Lukas, K.Y. Lee, H. Ghezel-Ayagh, Control Engineering Practice 2002, 10, 197-206.

[8] B. Kang, J. Koh, H. Lim, Journal of Power Sources 2001, 94, 51-62.

[9] C. Shen, G. Cao, X. Zhu, X. Sun, Journal of Process Control 2002, 12, 831-839.

[10] X. Sun, G. Cao, X. Zhu, J. Intell. Robot. Syst. 2001, 31, 299-316.

[11] S. Cavallaro, S. Freni, Journal of Power Sources 1998, 76, 190-196

[12] Kaihu Hou, Ronald Hughes, Chemical Engineering Journal 2001, 82, 311328

[13] K. Hoo, D. Zheng, Chem. Engng. Sci. 2001, 56, 6683-6710.

[14] J. Baker, P. Christofides, Int. J. Control 2000, 73, 439-456.

[15] J. A. Atwell, B.B. King, Math. Comp. Modelling 2001, 33, 1-19. 
[16] P. Christofides, P. Daoutidis Comp. Chem. Engng. (Suppl.), 1996, 20, S1071-S1076.

[17] Loève, M., Probability Theory. Van Nostrand, Princeton, NJ, 1955.

[18] H.M. Park, D.H. Cho, Chemical Engineering Science, 1996, 51, 81-98 